\title{
Three-dimensional thermal boundary layer corrections for circular heat flux gauges mounted in a flat plate with a surface temperature discontinuity
}

\author{
M. Kandula, a,", G. F. Haddad ${ }^{\mathrm{b}}$, R.-H. Chen ${ }^{\mathrm{c}}$

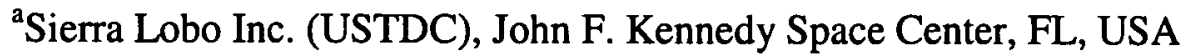

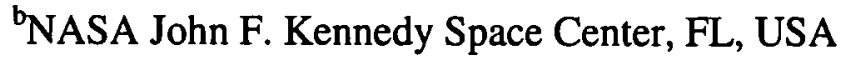 \\ ${ }^{c}$ Mechanical, Materials and Aerospace Engineering Department, \\ University of Central Florida, Orlando, FL, USA
}

\begin{abstract}
Three-dimensional Navier-Stokes computational fluid dynamics (CFD) analysis has been performed in an effort to determine thermal boundary layer correction factors for circular convective heat flux gauges (such as Schmidt-Boelter and plug type)mounted flush in a flat plate subjected to a stepwise surface temperature discontinuity. Turbulent flow solutions with temperature-dependent properties are obtained for a free stream Reynolds number of 1E6, and freestream Mach numbers of 2 and 4 . The effect of gauge diameter and the plate surface temperature have been investigated. The 3-D CFD results for the heat flux correction factors are compared to quasi-2D results deduced from constant property integral solutions and also 2-D CFD analysis with both constant and variable properties. The role of threedimensionality and of property variations on the heat flux correction factors has been demonstrated.
\end{abstract}

\footnotetext{
- Corresponding author. Fax: 3218674424

E-mail address: max.kandula-1@ksc.nasa.gov
} 
Key Words: Convective heat flux gauges, surface temperature discontinuity, threedimensional thermal boundary layers

\section{Nomenclature}

b $\quad\left(T_{w 1}-T_{a w}\right) /\left(T_{w 2}-T_{a w}\right)$

c sound speed, $\sqrt{\not R T}$

$c_{p} \quad$ specific heat at constant pressure

$h \quad$ heat transfer coefficient

$k \quad$ thermal conductivity

$L \quad$ reference plate length

$M \quad$ Mach number, $u_{\infty} / c$

$N u_{x} \quad$ local Nusselt number, $h_{x} x / k$

Pr Prandtl number

$q \quad$ heat flux, $h\left(T_{w}-T_{a w}\right)$

$R \quad$ gas constant; also radius of heat flux gauge

Re Reynolds number

$r \quad$ recovery factor

$S t_{x} \quad$ local Stanton number, $h_{x} /\left(\rho_{\infty} c_{p} u_{\infty}\right)=N u_{x} /\left(\operatorname{Re}_{x} \operatorname{Pr}\right)$

$T \quad$ temperature

$u, v, w$ velocities in the $x, y, z$ directions respectively

$x \quad$ streamwise direction

y lateral direction 
$z \quad$ direction normal to the plate surface

\section{Greek Symbols}

$\delta_{0} \quad$ hydrodynamic boundary layer thickness at the step

$\phi \quad\left(T_{w 1}-T_{w 2}\right) /\left(T_{w 1}-T_{a w}\right)$

$\gamma \quad$ ratio of specific heats (isentropic exponent)

$\rho$ density

$\mu \quad$ dynamic viscosity

$\tau_{w} \quad$ wall shear stress

$\zeta \quad\left(T_{w 2}-T_{w 1}\right) /\left(T_{w 2}-T_{a w}\right)$

\section{Subscripts}

0 stagnation

$1 \quad$ plate

2 gauge

A area-averaged

aw adiabatic wall (recovery)

$L \quad$ length-averaged

$x \quad$ local

wall

$\infty \quad$ freestream 


\section{Introduction}

Cylindrical flush mounted heat flux gauges (such as Schmidt-Boelter and plug type gauges) are frequently used on launch vehicles for measuring convective and radiative incident heat flux. The presence of the heat flux gauge necessarily alters the temperature and heat flux distribution on the gauge surface [1]. These gauges (normally metallic such aluminum, stainless steel, copper, etc.), when flush mounted in ablators or insulators of relatively low thermal conductivity (forming the vehicle surface), are subjected to wall temperatures considerably lower than that of the surrounding vehicle skin material (by several hundred degrees). On account of this surface temperature discontinuity (mismatch), heat exchange occurs between the gauge and the surrounding material by conduction, and the thermal boundary layer is altered (distorted), producing changes in the convective heat transfer coefficient from the fluid to the gauge surface. In convection, the thermal history of the boundary layer is carried with the fluid and affects the downstream heat transfer [1]. The changes in surface temperature and the departure of the heat transfer coefficient from the isothermal value demand that the heat flux measured by the calorimeter (gauge) deviates considerably from the true heat flux that would be measured if the gauge is formed of the same material as that of the insulation [2-3]. Experiments and analyses [4-10] have shown that a variable surface temperature distribution can produce a marked increase or decrease in the local and average convective heat transfer rates to a surface in laminar and turbulent flow.

Correction factors have been proposed in the past to account for the departure of the measured heat flux from the true heat flux. Rubesin [4] and Reynolds et al. [5-6] presented two-dimensional integral solutions for the local heat transfer coefficient in incompressible flow past a flat plate with zero pressure gradient and a stepwise surface temperature with the 
assumption of power law profiles for velocity and temperature. These solutions are based on the superposition of well-known unheated starting length solutions $[2,11]$. Recently, Mukerji et al. [12] extended the integral solution of Reynolds et al. [5,6] with an empirical correction (based on low speed data) to accommodate the effect of the viscous sublayer on the gauge surface (see Appendix A.3). Such a correction increases the influence of the temperature jump especially for small gauges, and offers a considerable improvement. On the basis of closed from integral solution due to Reynolds et al. [5,6], Westkaemper [13] derived a length-averaged heat transfer coefficient for the heat flux gauge.

Many simplifying assumptions are considered in the development of the integrals. The $1 / 7$ th power law solution is valid only far downstream of the step, as the profiles are developed at relatively large distances downstream of the step. Very near the temperature step, the thermal boundary layer is confined to the viscous sublayer. Artificial diffusivity in the sublayer (on account of $1 / 7$ th power law profiles throughout), which is the most important part of the boundary layer, produces error in the region immediately downstream of the step in temperature [11]. Also, the boundary layer equations may not strictly apply near the leading edge of the discontinuity. Thus the accuracy of the integral solution at distances within a few boundary layer thicknesses is questionable. It is known that the integral solution due to Reynolds et al. $[5,6]$ is known to underpredict experimental data by as much as 25 percent at $x / \delta_{0}=1.0$, and exceeds 30 percent for $x / \delta_{0}=0.25$ [12]. Another major deficiency of these integral solutions is connected with the assumption of constant thermal properties, which can lead to significant errors when the difference between the plate and the gauge surface temperatures becomes large, such as 
occurs at high Mach numbers encountered in flight conditions. In such circumstances solutions of the Navier-Stokes equations are necessary.

Kandula and Reinarts [14] carried out a 2-D Navier-Stokes CFD analysis with variable properties to investigate thermal boundary layer corrections for the local heat transfer coefficient. It was shown that the correction factor for convective heat transfer coefficient increases with the wall temperature. To account for the circular geometry gauge, a quasi-two dimensional analysis was also carried out, wherein at any lateral plane of the gauge, the flow is assumed quasi-two dimensional, so that area-averaged heat transfer correction factor incorporating 3-D effects approximately can be estimated. Comparisons were then presented between the quais-2 $\mathrm{D}$ results from CFD (variable properties) and the constant property integral solutions, signifying the importance of property variations.

The assumption of quasi-2D approximation can be in error as the size of the heat flux gauge is reduced in view of the preponderating effects of the three-dimensionality of the thermal boundary layer. In such circumstances, it is imperative to consider the full threedimensional thermal boundary layer effect on the heat flux correction factors. To the authors' knowledge, no such study has been reported previously.

In this report, three-dimensional Navier-Stokes simulation of the thermal boundary layer has been carried out for the plate-gauge system subjected to a stepwise surface temperature discontinuity. The effect of various relevant parameters on the correction factors has been studied. Comparisons of the 3D analysis with quasi-2D CFD results and integral solutions have also been performed. The effect of three-dimensionality of the thermal boundary layer and of property variations on the convective heat flux corrections for the gauges has been investigated. 


\section{Three-Dimensional CFD Analysis}

\subsection{Physical Assumptions}

The following physical assumptions are made in the CFD analysis.

a. The heat flux gauge is in steady state (water-cooled).

b. The gauge surface is idealized as isothermal. For example, a Schmidt-Boelter gauge is not strictly isothermal even when it is water cooled [15].

c. The external flow is uniform, and pressure gradient, surface curvature and separated flow are absent.

d. The Prandtl number of the fluid is taken as 0.7 .

e. Both the plate and the gauge surfaces are smooth, with the gauge flush-mounted into the plate.

\subsection{Grid System}

The overlapping grid system for the plate/gauge configuration is shown in Fig. 1a, indicating the plate grid ( $118 \times 72 \times 84$ size in the streamwise, lateral and normal directions)

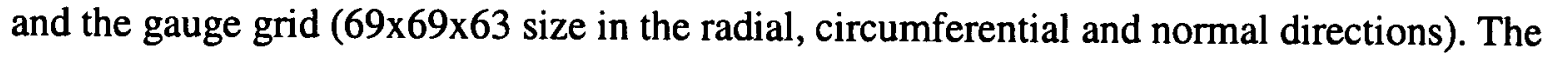
total number of grid points is about $9.9 \times 10^{5}$. Individual grids for the plate and the gauge are generated. The inter-grid communication is provided by the Pegasus code, Benek et al. [16]. Fig. 1b displays a side view of the grid. A plan view (partial) of the grid is provided in Fig. 1c.

An upstream inviscid plate length $(-0.5<x / L<0)$ is considered. The plate leading edge and trailing edges are located at $x / L=0$ and $x / L=2$ respectively, with the gauge surface centered at $x / L=1$. The grid normal extent is taken as $z / L=0.9$. For flow resolution 
purposes, the grid is clustered in the normal direction (near the wall) and in the axial direction (near the leading edge and near the surface temperature discontinuity).

\subsection{Flow Solution}

The flow solution (for density, velocity and temperature distribution) has been obtained by the OVERFLOW compressible Navier-Stokes CFD code $[17,18]$. A zonal two-equation $k-\omega$ SST (Shear Stress Transport) turbulence model due to Menter [19] has been considered. The $k-\omega$ model has been validated for boundary layers and free shear layers [20].

Freestream boundary conditions are applied at the inflow boundary $(x / L=-0.5)$, top boundary and at the lateral boundary away from the symmetry plane. Lateral symmetry is used at the symmetry plane. An extrapolation boundary condition is considered at the outflow $(x / L=2)$. Viscous wall condition is prescribed at the plate and the gauge surface. Isothermal conditions are specified for the plate surface $\left(T=T_{w 1}\right)$ and the gauge surface $\left(T=T_{w 2}\right)$.

The steady state solution is obtained by multi-gridding and local time-stepping. The algorithm used is diagonalized ADI (Alternating Direction Implicit), with central differencing for fluxes. Sutherland correlations are considered for the temperature dependence of viscosity and thermal conductivity of air [21,22].

\subsection{Flow and Geometry Parameters}

Solutions were obtained for a freestream Reynolds number of 1E6, and freestream Mach numbers of 2 and 4 . Values of the gauge radius to plate length ratio $R / L=0.005,0.01$, $0.025,0.05,0.075$ and 0.1 are considered. The freestream temperature is $288 \mathrm{~K}(519 \mathrm{R})$, and the gauge surface temperature is held at $333 \mathrm{~K}(600 \mathrm{R})$. The plate wall temperature is varied 
in the range $389 \mathrm{~K}$ to $1944 \mathrm{~K}$ ( $700 \mathrm{R}$ to $3500 \mathrm{R}$ ), and comprises values on either side of the adiabatic wall (recovery) temperature. The recovery temperature is computed from

$$
\frac{T_{a w}}{T_{\infty}}=1+r \frac{\gamma-1}{2} M^{2}
$$

where the turbulent recovery factor is obtained from

$$
r=\operatorname{Pr}^{1 / 3}
$$

With an assumed value of $\operatorname{Pr}=0.7$, the above formula yields a recovery temperature of $494 \mathrm{~K}$ for $M=2$, and $1111 \mathrm{~K}$ for $M=4$.

\section{Results and Comparison}

As indicated in the assumptions, all the results presented here with regard to CFD and the integral models are limited to uniform flow past a flat plate, where pressure gradient, surface curvature or separated flow are absent. The computations are performed on an 8processor (R-10000) SGI Origin-2000 machine.

\subsection{Solution Convergence}

Fig. 2 shows the convergence history for the solution residuals in a typical case. This residual history serves as a qualitative measure of the convergence to steady state, and suggests that convergence in residuals (several orders of magnitude drop in residuals) for the two grids is approached in about 2000 time iterations.

\subsection{Local Stanton number Distribution}

The local Stanton number distribution in the symmetry plane for a particular case is illustrated in Fig. 3a for $R / L=0.01$ and $M=4, T_{w 1} / T_{a w}=0.5$, and $T_{w 2} / T_{a w}=0.3$. A jump in the local Stanton number on the gauge surface $(0.99<x / L<1.01)$ is noted, as is to be expected. Downstream of the gauge, the recovery in the local Stanton number is established. 
The close agreement between the plate solution and the gauge solution in the overlapping region suggests that the overlapping grid scheme is satisfactory.

A schematic of the thermal boundary layer in the vicinity of the discontinuity for the symmetry plane is illustrated in Fig. 3b. The emergence of a new thermal boundary layer past the discontinuity is illustrated. Fig. $3 \mathrm{c}$ highlights the Stanton number distribution on the gauge surface. The extent of the three-dimensionality of the thermal boundary layer is evident near the leading edge and the trailing edge of the gauge.

\subsection{Heat flux correction factors}

The effect of variation of properties on the heat flux correction factors is illustrated in Fig. 4a for the case of $M=4$ and $R / L=0.01$. The constant property quasi-2D solutions for the integral method and the modified integral method [12] are compared with the quasi-2D CFD results for both constant and variable properties, and with the 3D CFD variable property solution. In the legend, CP stands for constant properties, and VP for variable properties. In general, a linear variation of $q_{2} / q_{1}$ with $\phi$ is noted, as predicated by the integral result of Eq. [A.5]. It is seen that the modified integral solution shows better agreement with the 2D CFD constant property solution. The variable property $2 \mathrm{D}$ CFD solution falls between the constant property 2D CFD solution and the modified integral solution. The 2D CFD solutions reveal that as the plate temperature to recovery temperature difference increases, the effect of the property variation becomes more pronounced.

Fig. 4a also suggests that the 3D CFD solution for the heat flux correction factor exceeds that obtained by quasi-2D CFD solution. The effect of three dimensionality is seen to increase with an absolute increase in $\phi$. The physical mechanism for the enhariced heat flux in three dimensions may be explained as follows. As the chord length of the gauge 
decreases away from the center-line, the quasi-2D assumption becomes more and more unrealistic, and the leading edge effect increases the overall 3D heat flux in the presence of a step. Data obtained by Mukerji et al. [12] under low turbulence (4 percent) and high turbulence (7-10 percent) freestream turbulence level suggest that spanwise transport of heat through turbulent diffusion is not an important effect for a 2-D heated element (increases the heat transfer rate by at most 5 percent).

Fig. $4 \mathrm{~b}$ shows the corrections factor variation as a function of $T_{w 1} / T_{a w}$ for $T_{w 1} / T_{a w}<1$. The results reveal that the deviation between 2D and 3D CFD increases as the wall temperature increases, indicating the importance of three-dimensional thermal boundary layer. The modified integral solution is seen to offer a considerable improvement over the integral solution when compared with the CFD results.

Comparisons for the correction factors for $M=0.2$ and $R / L=0.01$ are presented in Fig. 5. The general trends are seen to be similar to those expressed in Fig. 4a. A direct comparison between 3D CFD results at $M=4$ and $M=2$ is displayed in Fig. 6, showing the effect of Mach number on the heat flux correction factors. The results reveal that the correction factor increases as the Mach number increases, as is to be expected owing to the role of compressibility and property variations. It is expected that the agreement would be closer if the values of $T_{w 2} / T_{a w}$ were kept constant in both cases.

Calculations for $M=4$ at $R / L=0.1$ (Fig. 7) indicate that the heat flux correction factors are smaller than those for $R / L=0.01$. Although the trends are similar those for $R / L=0.01$, it is observed that there is no appreciable difference between the 3D and the 2D CFD results, suggesting that the effect of three dimensionality becomes negligible for $R / L=0.1$. 
The heat flux correction factors for $M=4$ at $R / L=0.005$ are depicted in Fig. 8 .

At $R / L=0.005$, the heat flux corrections factors are found to be higher than those

for $R / L=0.01$, as is to be expected. Again the modified integral solution agrees better with the 2D CFD solution as compared with the original integral solution. It is remarkable that the 3D CFD results are smaller than those from 2D values in the range of $T_{w 1} / T_{a w}$ from 0.3 to 0.45 . The physical basis for this behavior is not clear.

\subsection{Comparison with test data}

In an effort to validate the predictions, comparisons are made with limited test data recently reported by Strobel et al. [23] at $M=4$ and $R e=1.2 \mathrm{E} 6$. The data were obtained from ground tests at NASA Marshall hot gas facility (HGF) employing hydrogen-air combustion. Heat flux data were obtained from Schmidt-Boelter gauges (4.76 mm diameter, aluminum) supplied by AEDC (Arnold Engineering Development Center), and reference thin-skin calorimeters ( $2.54 \mathrm{~mm}$ thick and $114 \mathrm{~mm}$ diameter; $17-4 \mathrm{PH}$ stainless steel) with the latter designed to eliminate 2D conduction. A range of heating conditions can be produced by varying combustor pressure and temperature. The test section is comprised of a flat plate (12.7 $\mathrm{mm}$ thick, $304.8 \mathrm{~mm}$ wide, and $501.7 \mathrm{~mm}$ long). Both bare steel plate and steel plate covered with acusil TPS (Thermal Protection System) insulating material are considered. The heat flux gauges are not water-cooled. Instead, the plate-gauge system is operated in dynamic (transient) mode. The measured surface temperatures suggest that the temperaturetime history becomes quasi-steady in a few seconds (the test duration is about $20 \mathrm{sec}$ ).

The thin skin heat flux is found to be comparable regardless of the surrounding material (steel or acusil). There are small discrepancies between heat flux gauge output and thin skin response when gauges are mounted in stainless steel. However large discrepancies are noted 
when the gauges are mounted in acusil on account of changes in the thermal boundary layer. The original data are reported in terms of cold wall heat flux $\left(T_{w}=300 \mathrm{~K}\right)$, and are converted to hot wall heat flux. Considering uncertainties in surface temperature measurement, material properties, and dimensions, and errors associated with lateral heat conduction loss, the measured heat flux data are believed to be within about seven percent accuracy.

Fig. 9 shows the variation of $q_{2} / q_{1}$ as a function of $R / L$ as predicted by various theories and their comparison with test data. The data at 10.2 atm stagnation pressure correspond to wall temperatures of $T_{w 1} / T_{a w}=0.508$ and $T_{w 2} / T_{a w}=0.303$, and those at $13.93 \mathrm{~atm}$ stagnation pressure correspond to $T_{w 1} / T_{a w}=0.515$ and $T_{w 2} / T_{a w}=0.296$. The predictions suggest that $q_{2} / q_{1}$ decreases with an increase in the value of $R / L$. The 3D CFD results are higher than 2D CFD values, and the deviation increases with a decrease in $R / L$. The test data corresponding to $R / L=0.01$ and 0.025 are seen to lie between the 2D CFD and 3D CFD results. In general the CFD results exceed considerably the constant property integral solution. For the range of conditions considered here, the integral solution of $[5,6]$ is within 5-25 percent lower than the CFD results. The modified integral solution [12] shows better agreement with the data and the CFD solutions, being within about 10 percent lower than the CFD. These comparisons thus tend to highlight the importance of the three dimensional boundary layer effects.

Referring to the accuracy of the turbulence model employed here, the two-equation $k-\omega$ model has been widely incorporated in 3D CFD codes and validated for complex threedimensional configurations (for example, see Refs. 28 and 29). Calculations by the authors for local Stanton number for an isothermal flat plate at $\operatorname{Re}=10^{6}$ and $\mathrm{M}=0.2$, the model is accurate within 2 to 6 percent of standard correlations over a wide range of temperatures. Thus for 
boundary layer type flows considered here (where pressure gradients and flow separation are absent), it is estimated that the uncertainty in the heat flux correction factor due to turbulence model is about 6 percent.

\section{Conclusion}

The three dimensional Navier-Stokes solution of the heat flux gauge subjected to a surface temperature discontinuity has led to some insight into the uncertainty in heat flux measurement. It has been shown that the heat flux correction increases with an increase in plate temperature relative to the gauge temperature. It also increases with decreasing value of the gauge radius, and is relatively weakly dependent on the flow Mach number. In general, the effect of variable properties becomes significant with an increase in the temperature differential between the plate and the gauge. The results suggest that the effect of threedimensionality tends to increase the heat flux correction considerably above the integral predictions. The modified integral correlation accounting for the sublayer effect performs much better than the integral solution. For relatively large values of $R / L$ (say $R / L=0.1$ or above), the three dimensional thermal boundary layer effects become insignificant. For relatively small values of $R / L(=0.005)$, there is seen to be a crossover point between the 3D CFD and 2D CFD results.

Comparisons with limited test data available agree favorably with the three dimensional thermal boundary layer results, and confirm the importance of three dimensionality of the heat flux gauge and of property variations. Additional comparisons are recommended to further validate the accuracy of the model over a wider range of test conditions. As the heat 
flux corrections are generally huge, it can be concluded that the water-cooled heat flux gauges should never be considered for measurement of convective heat flux.

\section{Acknowledgements}

The authors would like to thank the reviewers, especially one of them, for valuable and insightful suggestions and criticism that considerably improved the manuscript. 


\section{Appendix A. Two-dimensional integral solutions}

\section{A.1 Local heat transfer coefficient}

Consider the flow past a two-dimensional flat plate with a surface temperature discontinuity

$$
\begin{aligned}
T_{w} & =T_{w 1} & & 0<x<L \\
& =T_{w 2} & & x \geq L
\end{aligned}
$$

With the aid of an integral method with assumed power law profiles for velocity and temperature, Rubesin [4] and later Reynolds, Kays and Klein [5,6] obtained an expression for the local heat transfer coefficient $h(x, L)$ in turbulent incompressible flow in the form

$$
\frac{h(x, L)}{h(x, 0)}=b+\zeta\left[1-\left(\frac{L}{x}\right)^{m_{1}}\right]^{m_{2}} \quad x>L
$$

where

$$
b=\left(T_{w 1}-T_{a w}\right) /\left(T_{w 2}-T_{a w}\right), \quad \zeta=\left(T_{w 2}-T_{w 1}\right) /\left(T_{w 2}-T_{a w}\right)
$$

Here, $h(x, 0)$ denotes the local heat transfer coefficient on an isothermal plate with constant wall temperature $T_{w 1}$. The exponents $m_{1}$ and $m_{2}$ are $39 / 40$ and $-7 / 39$ respectively from Rubesin [4], and 9/10 and -1/9 from Reynolds [5, 6], which is valid over a wider range of Reynolds number. For a single step in temperature $\left(T_{w 1}=T_{\infty}, T_{w 2}=T_{w}\right)$, the solution due to Reynolds et al. $[5,6]$ is expressed by

$$
S t_{x}=0.0287 \operatorname{Re}_{x}^{-0.2} \operatorname{Pr}^{-0.4}\left[1-(\zeta / x)^{9 / 10}\right]^{-1 / 9}
$$

The corresponding local heat flux ratio is expressed by [9]

$$
\frac{q_{2}(x, L)}{q_{1}(x)}=1-\phi\left[1-(L / x)^{m_{1}}\right]^{m_{2}}
$$

where

$$
\phi=\left(T_{w 1}-T_{w 2}\right) /\left(T_{w 1}-T_{a w}\right)
$$

and

$$
q_{1}=h(x, L)\left(T_{w 1}-T_{a w}\right), \quad q_{2}=h(x, 0)\left(T_{w 2}-T_{a w}\right)
$$


For very small values of $L / x, q_{2} / q_{1} \rightarrow 1-\phi$, which depends on temperatures only, and independent of geometric parameters [9].

\section{A.2 Length averaged heat transfer coefficient}

Based on the integral solution of $[5,6]$, Westkaemper [12] derived a length-averaged heat transfer coefficient $\bar{h}_{L}$ over the heat flux gauge as:

$$
\eta_{L}=\frac{\bar{h}_{L}}{h\left(\frac{W+L}{2}, 0\right)}=b F(L / W)+\zeta H(L / W)
$$

where $\bar{h}_{L}=\bar{h}(W, L)$ is given by

$$
\bar{h}_{L}=\frac{Q}{(W-L)\left(T_{w 2}-T_{a w}\right)}, \quad Q=\int_{L}^{W} q(x) d x=\int_{L}^{W} h(x, L)\left(T_{w 2}-T_{a w}\right) d x
$$

The factors $F$ and $H$ are defined by

$$
\begin{aligned}
& F=c_{k} \frac{5}{4} \frac{\left\lfloor 1-(L / W)^{4 / 5}\right\rfloor}{(1-(L / W))} \\
& H=c_{k} \frac{5}{4} \frac{(L / W)^{4 / 5}}{(1-L / W)}\left[(W / L)^{9 / 10}-1\right]^{8 / 9}-F(L / W)
\end{aligned}
$$

where $c_{k}=1$. Later, Knox [24] pointed out an error in Westkaemper's equations for $F$ and $H$. Considering that

$$
h\left(\frac{W+L}{2}, 0\right) \propto h(W, 0)\left(\frac{2}{1+L / W}\right)^{1 / 5}
$$

it was shown that the correction is provided by

$$
c_{k}=[2 /(1+L / W)]^{1 / 5}
$$




\section{A.3 Modified Integral Solution due to Mukerji et al. [12]}

Based on STA7 2D boundary layer finite difference code in conjunction with low speed data, Mukerji et al. [12] recently proposed the following correlation, as an extension of the integral solution of Reynolds et a. [5,6]:

$$
\begin{gathered}
S t(x)=0.0287 \operatorname{Re}_{x}^{-0.2} \operatorname{Pr}^{-0.4}\left\{\left[1-\left(\frac{\xi}{x}\right)^{9 / 10}\right]^{-1 / 9}+\phi\left(\xi, x, \operatorname{Re}_{\xi}\right)\right\} \\
\phi=\left(-0.0139 \ln \left(\operatorname{Re}_{\xi}\right)+0.246\right)\left(\frac{\xi}{x}\right)^{2} \frac{1}{\sqrt{1-(\xi / x)}}
\end{gathered}
$$

where

and represents a correction to the Reynolds correlation. For large values of $x / \delta_{0}$ (where $\delta_{0}$ represents the hydrodynamic boundary layer thickness at the location of the temperature step $\xi$, and $x$ the downstream distance from the step), this relation reduces to Eq. [A.4] due to Reynolds et al. $[5,6]$. The square root term controls the shape of the curve near the step. The correlation for local Stanton number agrees within 5 percent of STAN7 for $x / \delta_{0}=0.05$ to 10 , and $\operatorname{Re}_{\xi}=5 \times 10^{5}$ to $3 \times 10^{6}$. It predicts low turbulence intensity ( 0.4 percent) rectangular calorimetric data within two percent.

It may be pertinent to point out that the near-step region has been investigated in $[25,26]$ for a step change in surface temperature, and in [27] for a step change in surface heat flux.

\section{Appendix B. Quasi-two-dimensional extension}

Kandula and Reinarts [13] considered a quasi-two dimensional extension to approximately account for the cylindrical geometry of the heat flux gauge. At any lateral plane of the gauge, the flow is assumed quasi-two dimensional, so that an area-averaged heat flux correction factor $\bar{h}_{A}$ incorporating the three-dimensional effects can be estimated based 
on the two-dimensional results (both variable property CFD and constant property integral solutions):

$$
\eta_{A}=\bar{h}_{A} / h\left(\frac{W+L}{2}, 0\right)
$$

where $\quad \bar{h}_{A}=\frac{2}{\pi^{2}} \int_{y^{\prime}=0}^{R} \bar{h}_{L}\left(W^{\prime}, L^{\prime}\right)\left(2 x^{\prime}\right) d y^{\prime}, \quad x=R \cos \theta, \quad y=R \sin \theta$

Calculations suggest that the quantities $\bar{h}_{A}$ and $\bar{h}_{L}$ denoting quasi-2D and 2-D corrections do not appreciably differ from one another. For instance, for $R / L=0.01$ at $M=4, R=1 E 6, T_{w 1} / T_{a w}=1.6$, the quantity $\bar{h}_{A}$ is only $2.5 \%$ higher than $\bar{h}_{L}$. 


\section{References}

[1] T.E. Diller, Advances in heat flux measurements, In: J.P. Hartnett, T.F. Irvine (Eds.), Advances in Heat Transfer, Academic Press Inc., New York, 1993, pp. 279-368.

[2] E.R.G. Eckert, Analysis of Heat and Mass Transfer, Second ed., McGraw-Hill, New York, 1972.

[3] R.D. Neumann, Thermal instrumentation - A state-of-the-art review, WL-TR-96-2107, Wright Patterson Air Force Base, Ohio, 1996.

[4] M.W. Rubesin, The effects of an arbitrary surface temperature variation along a flat plate on the convective heat transfer, NACA TN 2345, April 1951.

[5] W.C. Reynolds, W.M. Kays, S.J. Klein, Heat transfer in the turbulent incompressible boundary layer II- Step -wall temperature distribution, NASA Memo 12-2-58W, December 1958.

[6] W.C. Reynolds, W.M. Kays, S.J. Klein, A summary of experiments on turbulent heat transfer from a nonisothermal flat plate, ASME J. Heat Transfer 82 (1960) 341-348.

[7] R.P. Taylor, P.H. Love, H.W. Coleman, M.H. Hosni, Heat transfer measurements in incompressible turbulent boundary layer with step wall temperature boundary conditions, ASME J. Heat Transfer 112 (1990) 245-247.

[8] R.J. Conti, Heat transfer measurements at Mach number of 2 in a turbulent boundary layer on a flat plate having a stepwise temperature distribution, NAS TN D-159, 1959.

[9] D.R. Hornbaker, D.L. Rall, Thermal perturbations caused by heat-flux transducers and their effect on the accuracy of heating rate measurements, ISA Trans. 3 (1964) 123-130. [10] R.C. Bachmann, J.T. Chambers, W.H. Giedt, Investigation of heat-flux measurements with calorimeters, ISA Trans. 4 (1965) 143-151. 
[11] W.M. Kays, M.E. Crawford, Convective Heat and Mass Transfer, second ed., McGrawHill, New York, 1980, pp. 214-215.

[12] D. Mukerji, J.K. Eaton, R.J. Moffat, Convective heat transfer near one-dimensional and two-dimensional wall temperature steps, ASME J. Heat Transfer 126 (2004) 202-210.

[13] J.C. Westkaemper, On the error in plug-type calorimeters caused by surface temperature mismatch, J. of Aerospace sciences (1961) 907-908.

[14] M. Kandula and T. Reinarts, Corrections for convective heat flux gauges subjected to a surface temperature discontinuity, AIAA-2002-3087, 2002.

[15] J. Hayes, A. Rougeux, The application of numerical techniques to model the response and integration of thermal sensors in wind tunnel models, AIAA-91-0063.

[16] J.A. Benek, P.G. Buning, J.L. Steger, A 3-D grid embedding technique, AIAA-85-1523CP, July 1985.

[17] D.C. Jespersen, T.H. Pulliam, P.G. Buning, Recent enhancements to OVERFLOW, AIAA-97-0644, January 1997.

[18] M. Kandula, P.G. Buning, Implementation of LU-SGS algorithm and Roe upwinding scheme in OVERFLOW thin-layer Navier-Stokes code, AIAA-94-2357, 1994.

[19] F.R. Menter, Two-equation eddy viscosity turbulence model for engineering applications, AIAA Journal 32 (1994), 1598-1605.

[20] M. Kandula, D.C. Wilcox, An examination of $k-\omega$ turbulence model for boundary layers, free shear layers and separated flows, AIAA-95-2317, 1995.

[21] H. Schlichting, Boundary Layer Theory, Seventh ed., McGraw-Hill, New York, 1979. [22] F.M. White, Viscous Fluid Flow, Second ed., McGraw-Hill, 1991. 
[23] F. Strobel, J. Grudgen, T. Reinarts, G. Russell, The use of heat flux gauges for hot wall TPS applications, Space Technology and Applications International Forum (STAIF), February 13-17, 2005.

[24] E.C. Knox, A critique on correlating heat transfer results for temperature mismatch, Remtech Report RTN 158-02, March 1987.

[25] J. Kestin, L.N. Persen, The transfer of heat across a turbulent boundary layer at very high Prandtl numbers, Int. J. of Heat Mass Trans. 5 (1962) 355-371.

[26] L.W.B. Browne, R.A. Antonia, Calculation of a turbulent boundary layer downstream of a step change in surface temperature, ASME J. Heat Transfer 101 (1979) 144-150.

[27] R.A. Antonia, H.Q. Danh, A. Prabhu, Response of a turbulent boundary layer to a step change in heat flux, J. Fluid Mechanics 80-part 1 (1977) 153-177.

[28] C.L. Rumsey, S.M. Rivers, J.H. Morrison, Study of CFD variation on transport configurations from the second drag-prediction workshop, AIAA-2004-0394.

[29] Y.-L. Lin, T.I.-P. Shih, Film cooling of a cylindrical leading edge with injection through rows of compound-angle holes, ASME J. Heat Transfer 123 (2001) 645-654. 


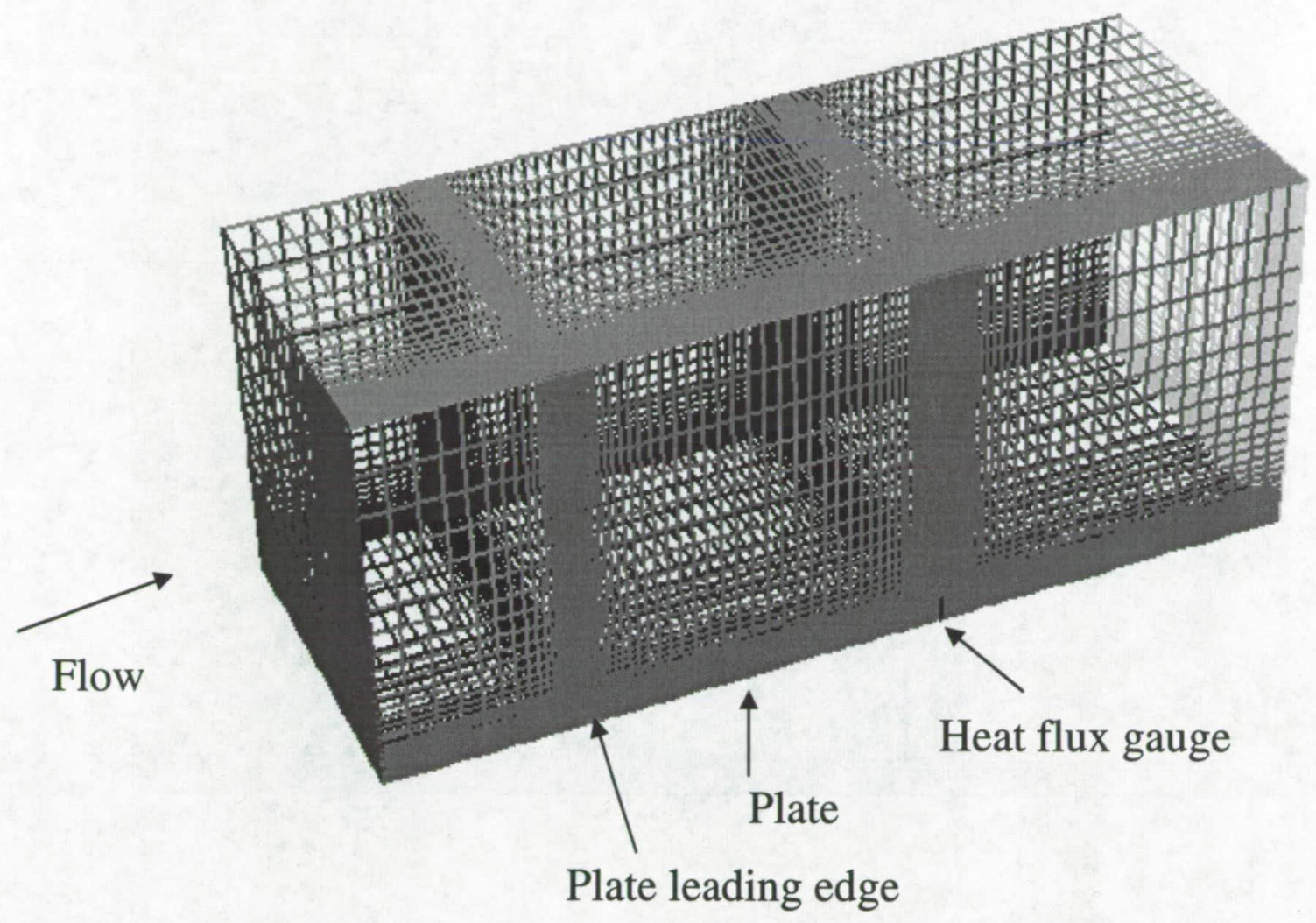

Fig. 1a. Overall view of the 3-dimensional grid system.

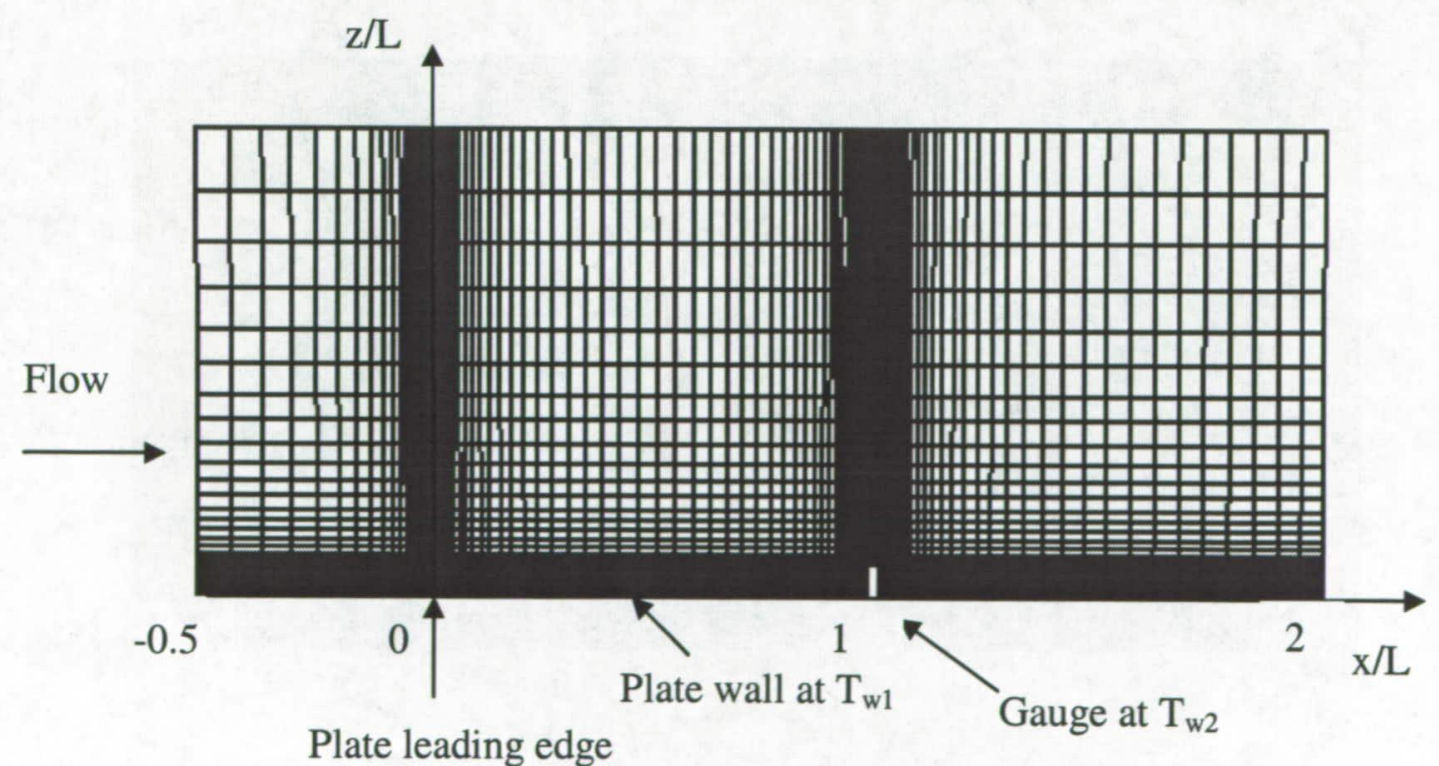

Fig. 1b. Side view of the grid system. 


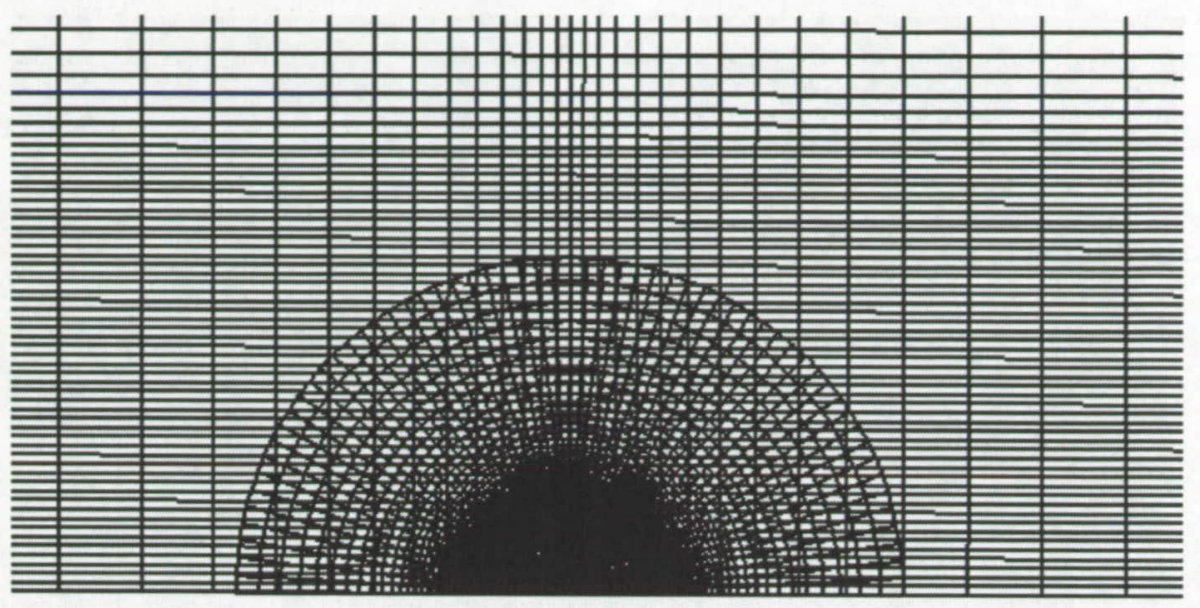

Fig. 1c. Plan view of the grid system in the neighborhood of the gauge.

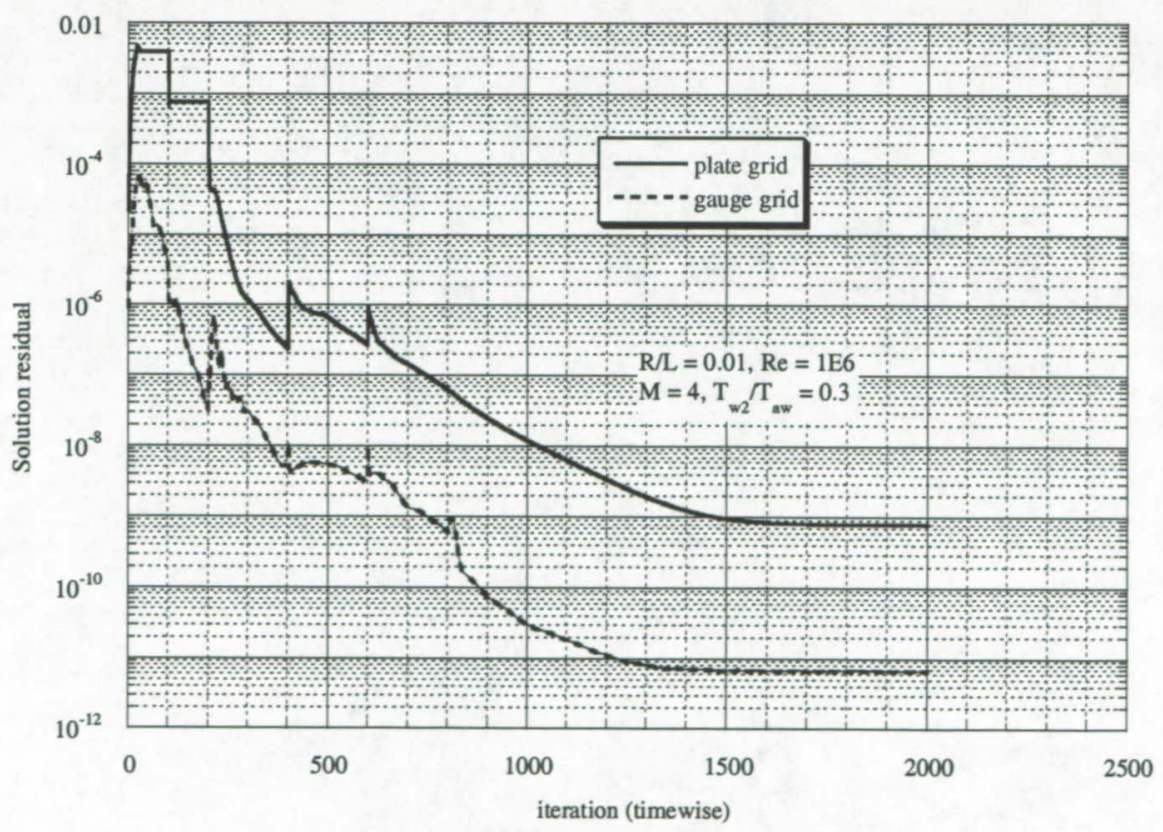

Fig. 2 Convergence history of solution residuals. 


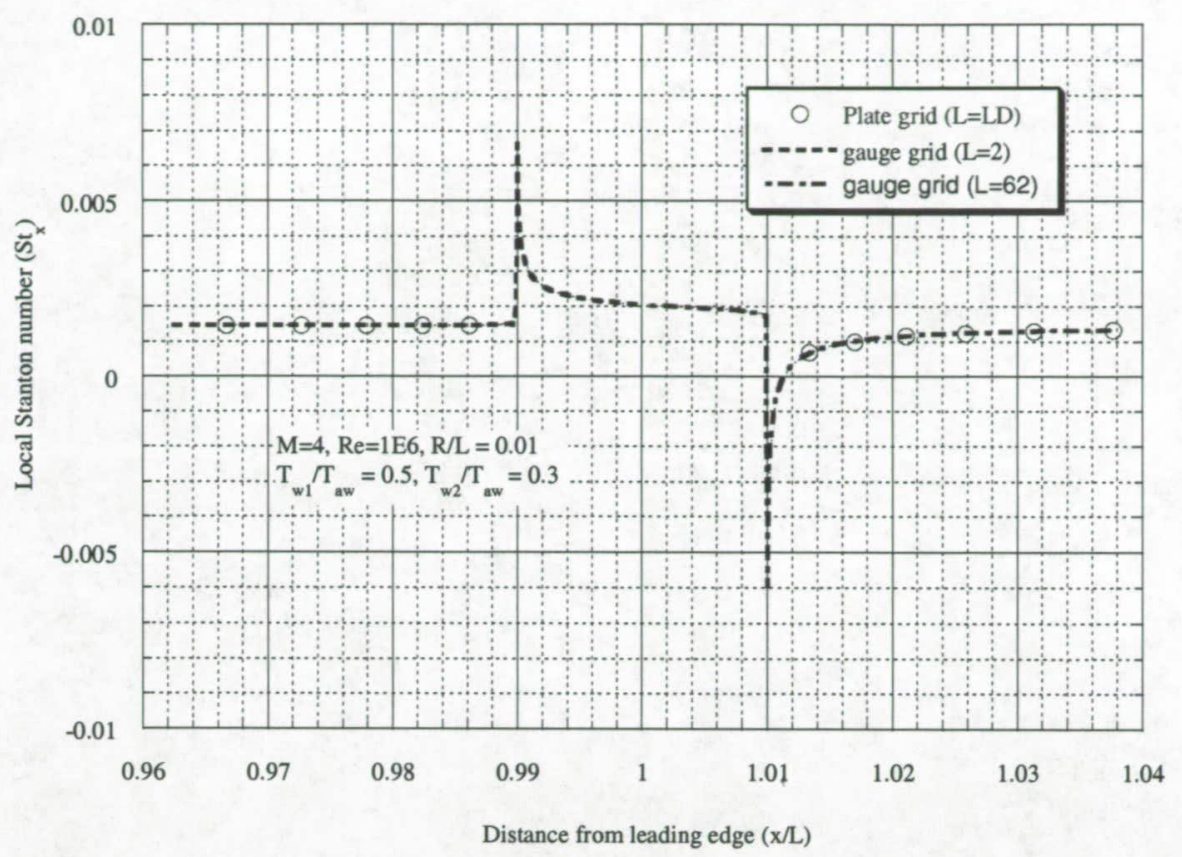

Fig. 3a. Local number Stanton number distribution in the symmetry plane.
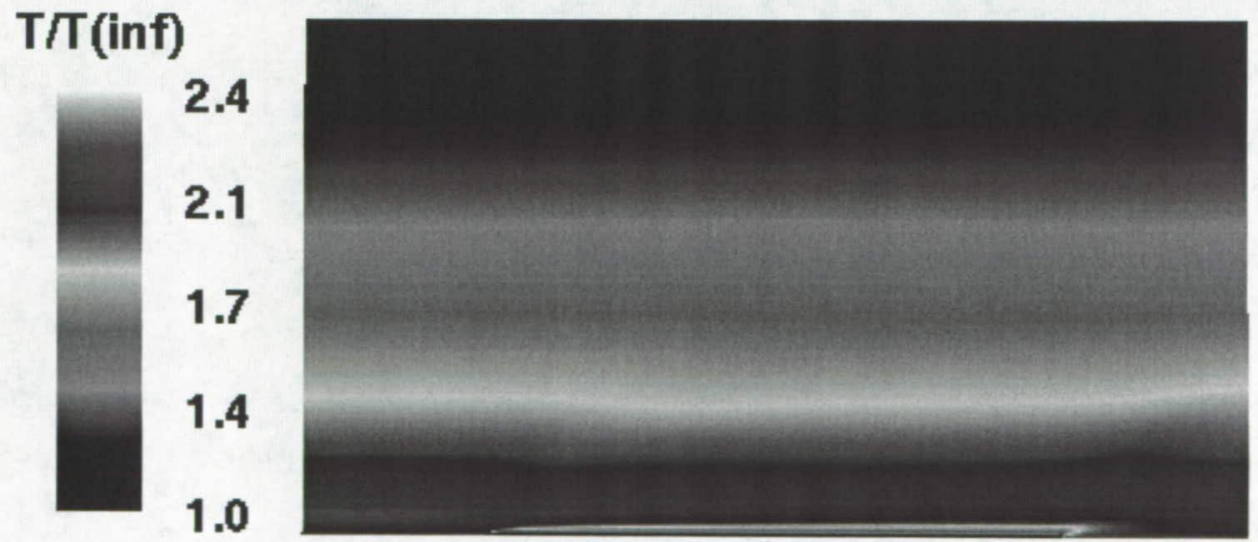

Fig. 3b Thermal boundary layer alteration near the temperature discontinuity 


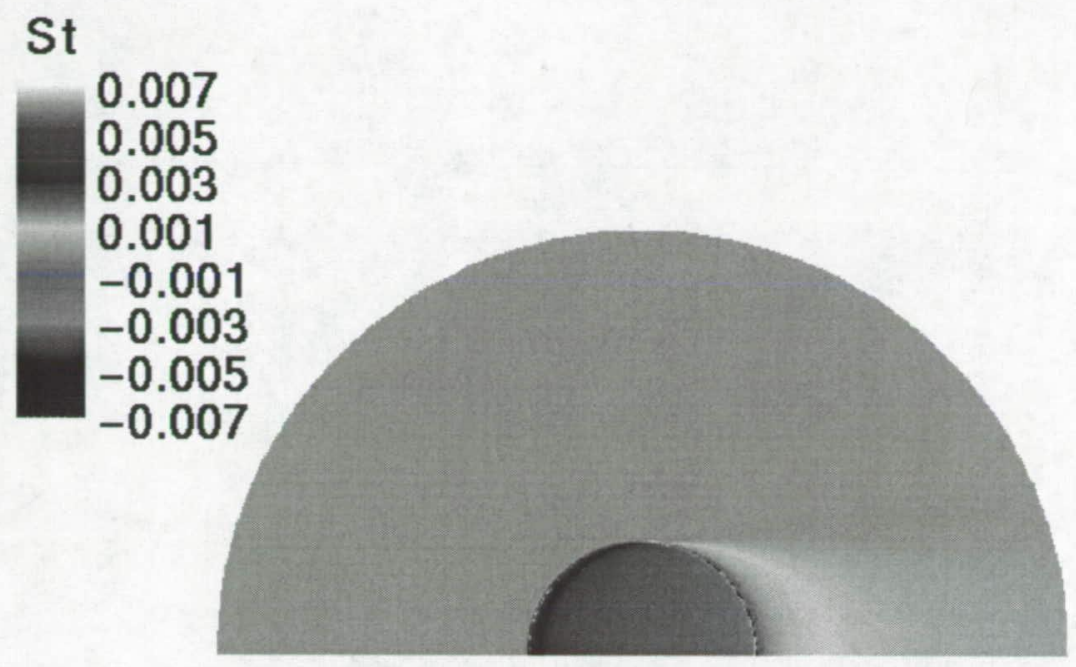

Fig. 3c Stanton number distribution on the gauge surface.

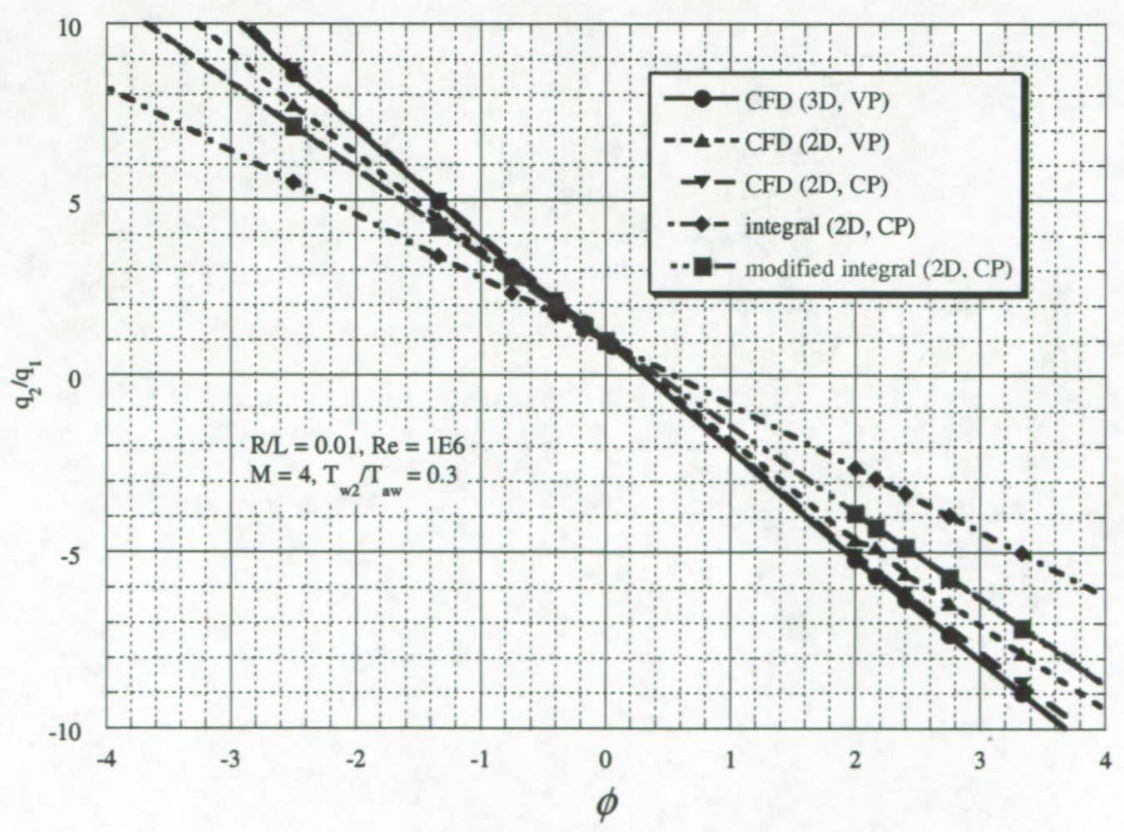

Fig. $4 \mathrm{a}$ Comparison of heat flux correction factor for $\mathrm{M}=4$ and $\mathrm{R} / \mathrm{L}=0.01$. 


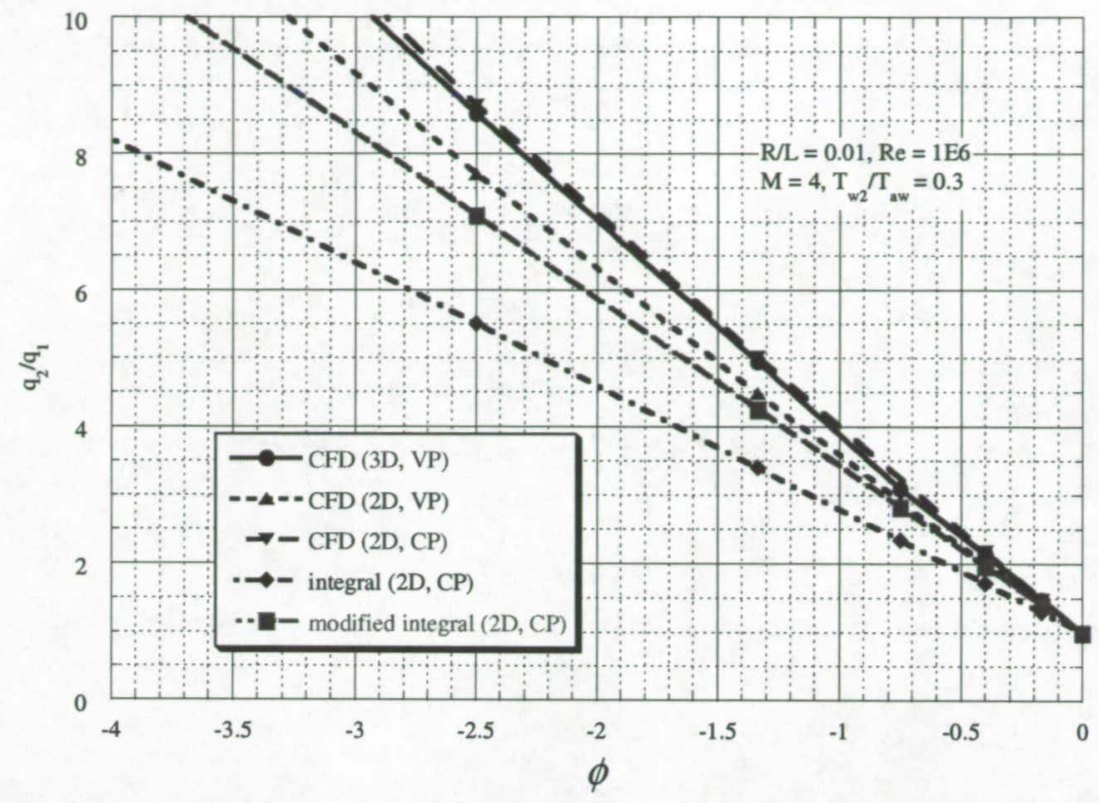

Fig. $4 b$ Comparison of heat flux correction factor for $M=4$ and $R / L=0.01$ (contd.).

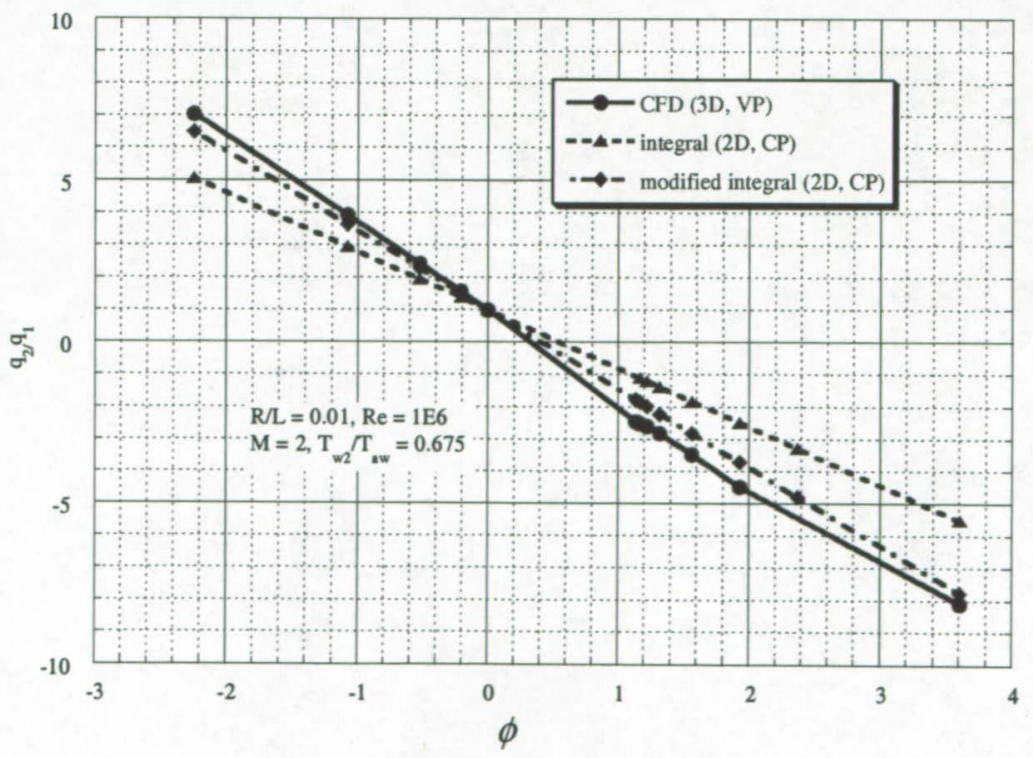

Fig. 5 Comparison of heat flux correction factor for $\mathrm{M}=2$ and $\mathrm{R} / \mathrm{L}=0.01$. 


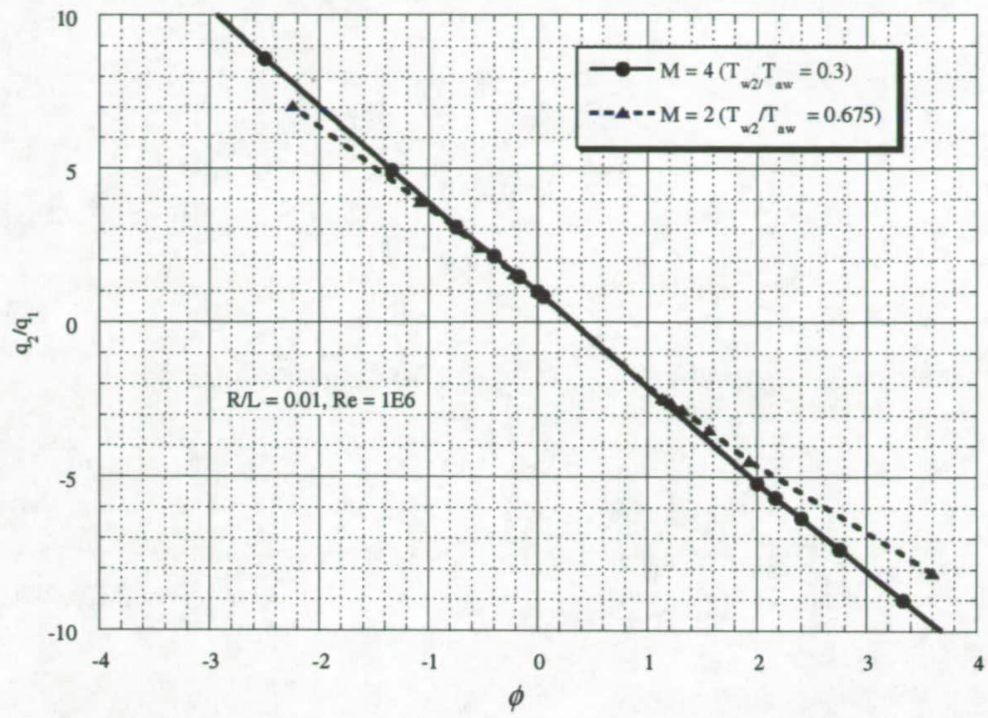

Fig. 6 Comparison of heat flux correction factor from 3D CFD for $\mathrm{M}=2$ and 4 At $\mathrm{R} / \mathrm{L}=0.01$.

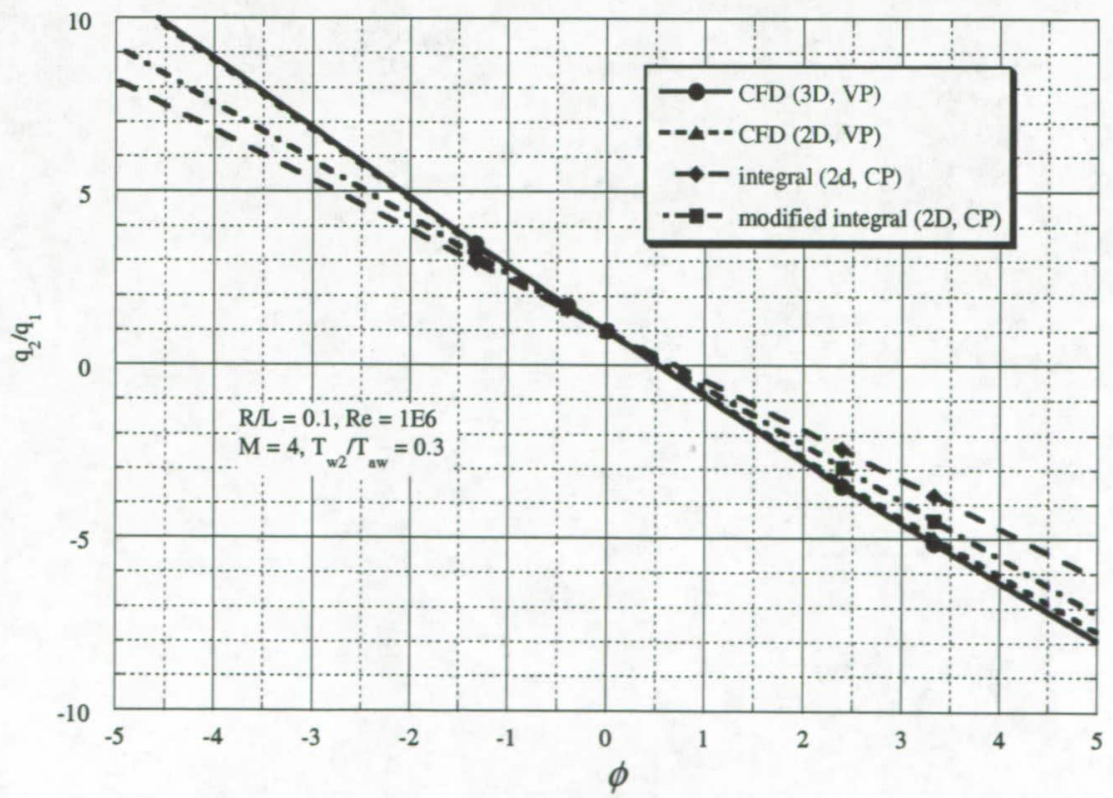

Fig. 7 Comparison of heat flux correction factor for $\mathrm{M}=4$ at $\mathrm{R} / \mathrm{L}=0.1$. 


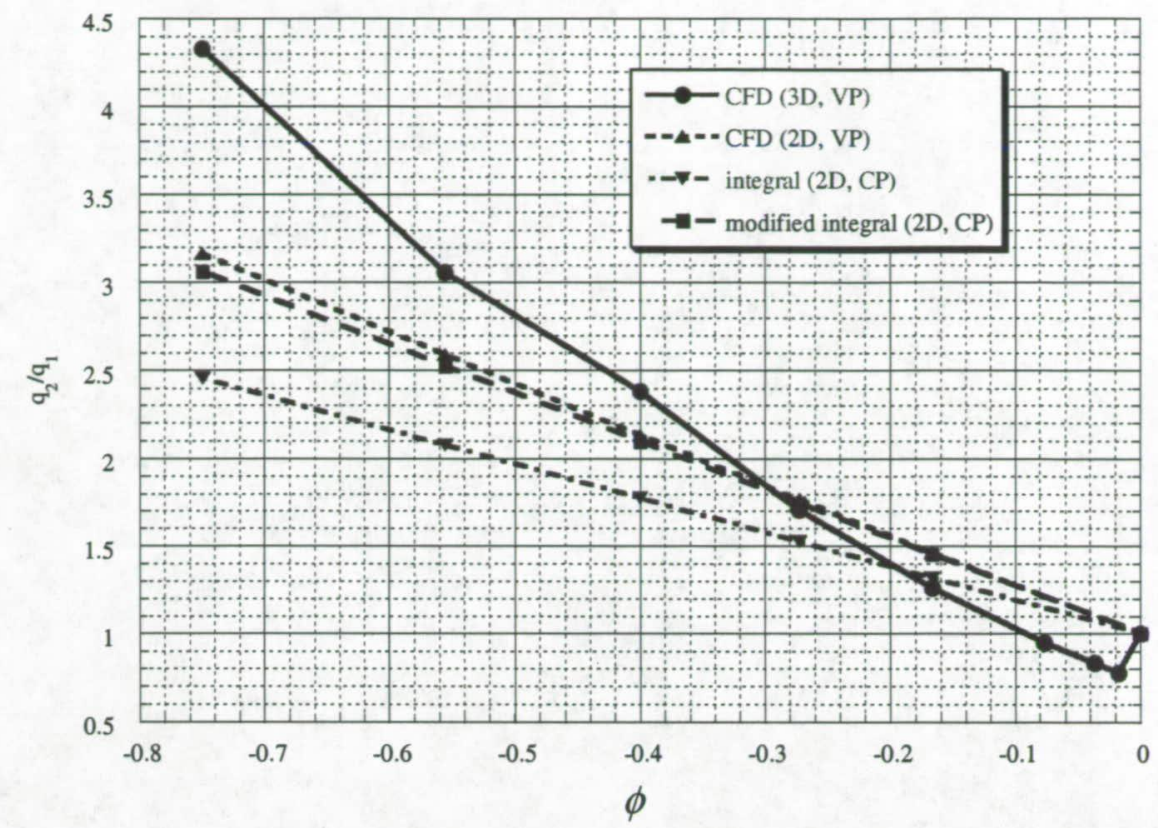

Fig. 8 Comparison of heat flux correction factor for $\mathrm{M}=4$ at $\mathrm{R} / \mathrm{L}=0.005$.

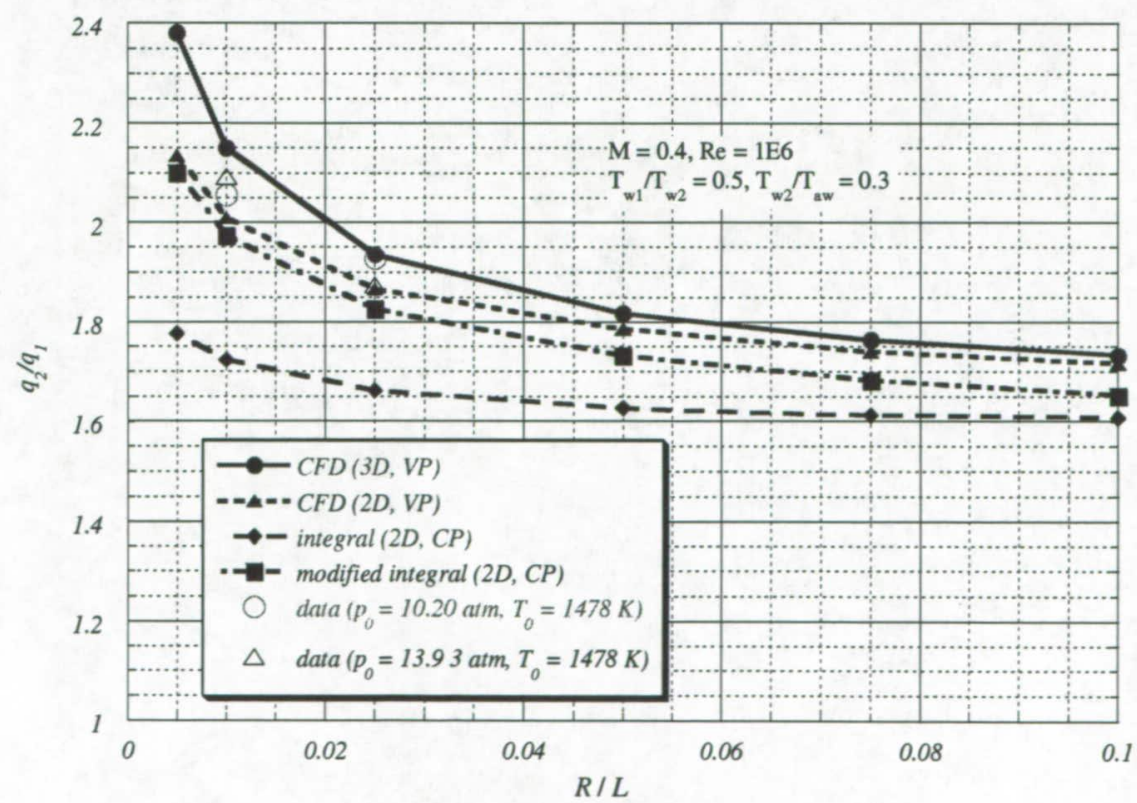

Fig. 9 Comparison of heat flux correction factors with test data for $\mathrm{M}=4$. 